\title{
Some Fixed Point Results in 2-Metric Spaces
}

\author{
Amalendu Choudhury ${ }^{1, *}$, T. Som ${ }^{2}$ \\ ${ }^{1}$ Department of Mathematics and Statistics Haflong Govt. College, Haflong, Dima Hasao Assam. Pin, 788819, India \\ ${ }^{2}$ Department of Applied Mathematics Indian Institute of Technology (BHU), Varanasi, 221005, India
}

\begin{abstract}
The present paper deals with few fixed point results for mappings satisfying some generalized contractive type inequality condition in 2-metric spaces, wh ich generalize the results of Rhoades (1979) and Gahler (1963), Iseki (1975), Iseki et.al (1976) in turn. The inqualities involve a rational type of terms given by $\left[\frac{\rho(\mathrm{x}, \mathrm{f}(\mathrm{x}), \mathrm{a})+\rho(\mathrm{y}, \mathrm{f}(\mathrm{x}), \mathrm{a})}{2}\right]$, $\left[\frac{\rho(\mathrm{x}, \mathrm{f}(\mathrm{y}), \mathrm{a})+\rho(\mathrm{y}, \mathrm{f}(\mathrm{x}), \mathrm{a})}{2}\right],\left[\frac{\rho(\mathrm{x}, \mathrm{g}(\mathrm{y}), \mathrm{a})+\rho(\mathrm{y}, \mathrm{f}(\mathrm{x}), \mathrm{a})}{2}\right],\left[\rho\left(\mathrm{x}, \mathrm{f}_{\mathrm{j}}^{\mathrm{mj}}(\mathrm{y}), \mathrm{a}\right)+\rho\left(\mathrm{y}, \mathrm{f}_{\mathrm{i}}^{\mathrm{mi}}(\mathrm{x}), \mathrm{a}\right)\right] / 2$ etc under max composition.
\end{abstract}

Keywords 2-Metric Space, Self Mapping, Generalized Contraction, Fixed Point

\section{Introduction}

The concept of a 2- metric space was initially given by Gahler ([2],[3]) during 1960's. Then about a decade after during 1970's some basic fixed point results in such spaces have been established by Iseki ([4], [5]). There after some fixed point results are obtained in such spaces by Khan et al [6], Rhoades [7] and many others extending the fixed point results for contractive mappings from metric space to 2metric space. In this subsection we give some preliminary definitions and results of aforesaid authors.

Definition 1.1 A 2-metric space is a space $\mathrm{X}$ in which, for each triple of points $a, b, c$ there exists a real valued non negative function satisfying:

(1) For each pair of points $a, b, a \neq b$ of $X$, there exists $a$ point $\mathrm{c} \varepsilon \mathrm{X}$ such that $\rho(\mathrm{a}, \mathrm{b}, \mathrm{c}) \neq 0$.

(2) $\rho(a, b, c)=0$ when at least two of the points are equal. (3) $\rho(a, b, c)=\rho(a, c, b)=\rho(b, c, a)$ and (4) $\rho(a, b, c) \leq \rho(a, b$, d) $+\rho(a, d, c)+\rho(d, b, c)$

Definition 1.2 A sequence $\left\{x_{n}\right\}$ of $X$ is called Cauchy sequence if

$\lim \rho\left(x_{n}, x_{m}, a\right)=0$ for all a $\varepsilon X$.

Definition 1.3 A sequence $\left\{x_{n}\right\}$ in $X$ is convergent and $x \varepsilon$ $\mathrm{X}$ is the limit of this

sequence if $\rho\left(x_{n}, x, a\right)=0$ for each a $\varepsilon X$.

Theorem 1.1 (Rhoades[7]) Let $X$ be a complete 2-metric Space, $\mathrm{f}: \mathrm{X} \rightarrow \mathrm{X}$ satisfying: there exists a $\mathrm{h}, 0 \leq \mathrm{h}<1$ such

$\rho(f(x), f(y), a) \leq h \max \{\rho(x, y, a), \rho(x, f(x), a), \rho(y, f(y), a)$, $\rho(\mathrm{x}, \mathrm{f}(\mathrm{y}), \mathrm{a}), \rho(\mathrm{y}, \mathrm{f}(\mathrm{x}), \mathrm{a})\}$

that for each $\mathrm{x}, \mathrm{y}, \mathrm{a} \varepsilon \mathrm{X}$

* Corresponding author:

choudhury.amalendu@y ahoo.com (Amalendu Choudhury)

Published online at http://journal.sapub.org/ajms

Copyright (C) 2012 Scientific \& Academic Publishing. All Rights Reserved
Then $\mathrm{f}$ possesses a unique fixed point $\mathrm{z}$ and $\lim \mathrm{f}^{\mathrm{n}}\left(\mathrm{x}_{0}\right)=\mathrm{z}$ for each $\mathrm{x}_{0} \& \mathrm{X}$.

Theorem 1.2 (Rhoades[7]) Let $f$ and $g$ be mappings of a complete 2- metric space $\mathrm{X}$ into itself satisfying

$\rho(\mathrm{f}(\mathrm{x}), \mathrm{g}(\mathrm{y}), \mathrm{a}) \leq \mathrm{h} \max \{\rho(\mathrm{x}, \mathrm{y}, \mathrm{a}), \rho(\mathrm{x}, \mathrm{f}(\mathrm{x}), \mathrm{a}), \rho(\mathrm{y}, \mathrm{g}(\mathrm{y}), \mathrm{a})$, $\rho(y, f(x), a), \rho(x, g(y), a)\}$

for all $\mathrm{x}, \mathrm{y} \varepsilon \mathrm{X}, \mathrm{h}$ a fixed constant satisfying $0 \leq \mathrm{h}<1$. Then $f$ and $g$ have a common fixed point $\mathrm{z}$.

The orem 1.3 (Rhoades [7]) Let $X$ be a complete 2- metric space, $\left\{\mathrm{f}_{\mathrm{n}}\right\}, \mathrm{n}=1,2, \ldots$ a sequence of mapping $\mathrm{f}_{\mathrm{n}}: \mathrm{X} \rightarrow \mathrm{X}$, suppose there exists a sequence of non negative integers $\left\{\mathrm{m}_{\mathrm{n}}\right\}$ and a number $\mathrm{h}, 0 \leq \mathrm{h}<1$ such that, for all $\mathrm{x}, \mathrm{y} \in \mathrm{X}$ and every pair $i, j, i \neq j$ and satisfying

$\rho\left(f_{i}^{\text {mi }}(x), f_{j}^{m j}(y), a\right) \leq h \quad \max \quad\{\rho(x, y, a)$, $\left.\rho\left(\mathrm{x}, \mathrm{f}_{\mathrm{i}}^{\mathrm{mi}}(\mathrm{x}), \mathrm{a}\right), \rho\left(\mathrm{y}, \mathrm{f}_{\mathrm{j}}^{\mathrm{mi}}(\mathrm{y}), \mathrm{a}\right), \rho\left(\mathrm{y}, \mathrm{f}_{\mathrm{i}}^{\mathrm{mi}}(\mathrm{x}), \mathrm{a}\right)\right\}$

Then the mappings $\left\{f_{n}\right\}$ have a unique common fixed point.

\section{Objective}

The main objective of the paper is to establish few fixed point results in 2-metric space involving terms like $\left[\frac{\rho\left(x_{s} f(x), a\right)+\rho(y, f(x), a)}{2}\right],\left[\frac{\rho\left(x_{s} f(y), a\right)+\rho(y, f(x), a)}{2}\right]$,
$\left[\frac{\rho\left(x_{,} g(y), a\right)+\rho(y, f(x), a)}{2}\right], \quad\left[\rho\left(x, f_{j}^{m j}(y), a\right)+\right.$
$\left.\rho\left(y, f_{i}^{m i}(x), a\right)\right] / 2$ etc in the inequality condition under max composition, which is more general than the inequality condition used by previous authors.

\section{Method}

The usual fixed point analysis methods for 2-metric space as used by earlier authors Gahler, Rhoades, Iseki has been 
used to prove our generalizations.

\section{Main Results}

In this paper few fixed point results are obtained for some generalized contractive mappings involving rational type terms in a 2- metric space. Taking a clue from Theorem 1.1, our first result goes as follows:

The orem 4.1 Let $X$ be a complete $2-$ metric space, $f: X$ $\rightarrow \mathrm{X}$ satisfying : there exists a $\mathrm{h}, 0 \leq \mathrm{h}<1$ such that for each $\mathrm{x}, \mathrm{y}, \mathrm{a} \varepsilon \mathrm{X}$

$\rho(f(x), f(y), a) \leq h \max \{\rho(x, y, a), \rho(x, f(x), a), \rho(y, f(y), a)$, $\left[\frac{p\left(x_{s} f(x)_{s} a\right)+p\left(y_{s} f(x)_{s} a\right)}{2}\right]$,

$\left.\left[\frac{p(x, f(y), a)+p(y, f(x), a)}{2}\right]\right\}$ (i) Then f possesses a unique fixed point $\mathrm{z}$.

Proof : Let $x_{0} \in X$ and define $\left\{x_{n}\right\}$ by

$\mathrm{x}_{\mathrm{n}+1}=\mathrm{f}\left(\mathrm{x}_{\mathrm{n}}\right), \mathrm{n}=0,1,2 \ldots$

From (i) we have,

$\rho\left(x_{n+1}, x_{m+1}, a\right)=\rho\left(f\left(x_{n}\right), f\left(x_{m}\right), a\right) \leq h \max \left\{\rho\left(x_{n}, x_{m}, a\right)\right.$, $\rho\left(x_{n}, x_{n+1}, a\right), \rho\left(x_{m}, x_{m+1}, a\right)$,

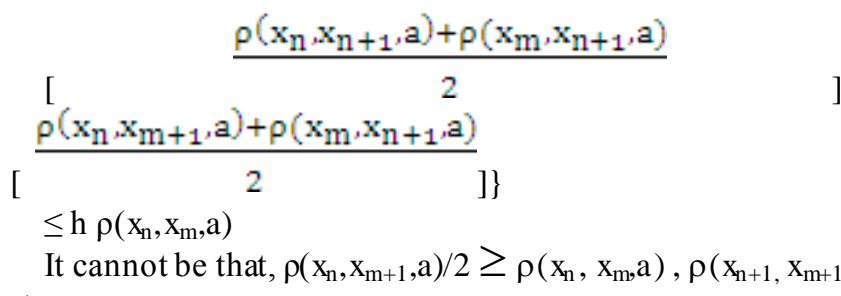

a)

Again ,

$\rho\left(x_{n}, x_{m+1}, a\right) \leq \rho\left(x_{n}, x_{m+1}, x_{n+1}\right)+\rho\left(x_{n}, x_{n+1}, a\right)+\rho\left(x_{n+1}, x_{m+1}, a\right)$

and we have, $\rho\left(x_{n}, x_{m+1}, x_{n+1}\right)=0$.

Thus,

$\rho\left(x_{n+1}, x_{m+1}, a\right) \leq h \rho\left(x_{n}, x_{m}, a\right)$

Similarly, $\rho\left(x_{n}, x_{m}, a\right) \leq h \rho\left(x_{n-1}, x_{m-1}, a\right)$

$\leq \mathrm{h}^{2} \rho\left(\mathrm{x}_{\mathrm{n}-2}, \mathrm{x}_{\mathrm{m}-2}, \mathrm{a}\right)$

$\leq \mathrm{h}^{\mathrm{n}} \rho\left(\mathrm{x}_{0}, \mathrm{x}_{\mathrm{k}}, \mathrm{a}\right)$

Therefore for integers $\mathrm{n}, \mathrm{m}$ where $\mathrm{n}>\mathrm{m} \geq 0$,

$\rho\left(x_{n}, x_{m}, a\right) \leq h^{n} \rho\left(x_{0}, x_{k}, a\right)$ (ii)

where $\mathrm{k}$ is a suitable integer satisfying $0<\mathrm{k} \leq \mathrm{m}$. using property 4 of definition 1

and (ii), we get

$\rho\left(x_{0}, x_{k}, a\right) \leq \rho\left(x_{0}, x_{k}, x_{1}\right)+\rho\left(x_{0}, x_{1}, a\right)+\rho\left(x_{1}, x_{k}, a\right)$

$\leq \rho\left(x_{0}, x_{k}, x_{1}\right)+\rho\left(x_{0}, x_{1}, a\right)+h \rho\left(x_{0}, x_{k}, a\right)$

$\leq \ldots \ldots \ldots \ldots \ldots \ldots$

$\leq \frac{1}{1-h} \rho\left(\mathrm{x}_{0}, \mathrm{x}_{1}, \mathrm{a}\right)$

Therefore, $\rho\left(x_{n}, x_{m}, a\right) \leq \frac{h^{n}}{1-h} \rho\left(x_{0}, x_{1}, a\right)$

So it can be easily shown that

$\rho\left(\mathrm{x}_{0}, \mathrm{x}_{\mathrm{k}}, \mathrm{x}_{1}\right)=0$, (see[7])

where $\mathrm{k}^{\prime}$ is a suitable integer satisfying $0 \leq \mathrm{k}^{\prime} \leq \mathrm{k}$. Therefore $\left\{x_{n}\right\}$ is Cauchy sequence, hence convergent. Let us consider the limit $\mathrm{z}$, using (i), for any a $\varepsilon \mathrm{X}$.

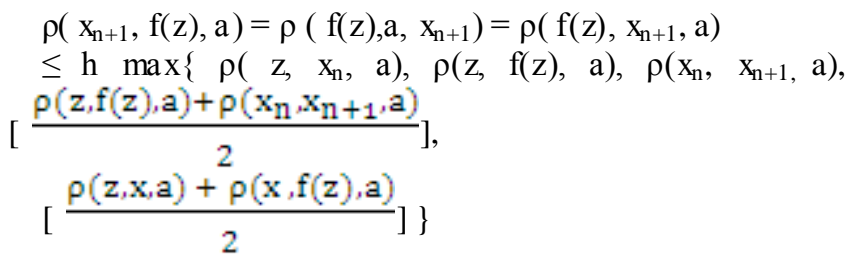

Taking the limit of both sides as $\mathrm{n} \rightarrow \infty$, we have, $\rho(\mathrm{z}, \mathrm{f}(\mathrm{z}), \mathrm{a}) \leq \mathrm{h} \rho(\mathrm{z}, \mathrm{f}(\mathrm{z}), \mathrm{a})$

which implies $z=f(z)$.

Suppose $z, w$ are fixed points of $f$. Then from (i), each a $\varepsilon$

$X$, we have

$\rho(\mathrm{z}, \mathrm{w}, \mathrm{a}) \leq \mathrm{h} \rho(\mathrm{z}, \mathrm{w}, \mathrm{a})$.

Since $0 \leq \mathrm{h}<1$, and using $\rho(\mathrm{a}, \mathrm{b}, \mathrm{c}) \neq 0$. So we get, $\mathrm{z}=\mathrm{w}$.

Therefore $\mathrm{f}$ has a unique fixed point $\mathrm{z}$.

Theorem 4.2 Let $X$ be a complete 2- metric space, $\left\{\mathrm{f}_{\mathrm{n}}\right\}$ a sequence of mappings of $X$ into $X$ with fixed points $z_{n}$, and $f$ a mappings of $X$ into $X$ satisfying

$\rho(f(x), f(y), a) \leq h \max \{\rho(x, y, a), \rho(x, f(x), a), \rho(y, f(y), a)$, $\rho(\mathrm{x}, \mathrm{f}(\mathrm{y}), \mathrm{a}), \rho(\mathrm{y}, \mathrm{f}(\mathrm{x}), \mathrm{a})\}$ (iii)

with fixed point $\mathrm{z}$, such that $\mathrm{f}_{\mathrm{n}} \rightarrow \mathrm{f}$ uniformly on $\left\{\mathrm{z}_{\mathrm{n}}: \mathrm{n}=\right.$ $1,2, \ldots\}$. Then $z_{n} \rightarrow z$.

Proof: Let $\varepsilon>0$. From the uniform convergence of $\left\{f_{n}\right\}$ on $\left\{z_{n}: n=1,2, ..\right\}$ there exists an integer $N$ such that for all $n \geq$ $\mathrm{N}$,

], $\quad \rho\left(\mathrm{f}\left(\mathrm{z}_{\mathrm{n}}\right), \mathrm{f}_{\mathrm{n}}\left(\mathrm{z}_{\mathrm{n}}\right), \mathrm{a}\right)<\frac{\varepsilon}{\mathrm{M}}$, for all $\mathrm{z}_{\mathrm{n}}$, where $\mathrm{M}=\frac{1}{1-\mathrm{h}}$

Now, $\rho\left(z_{n}, z, a\right)=\rho\left(f_{n}\left(z_{n}\right), f(z), a\right)$

$\leq \rho\left(f_{n}\left(z_{n}\right), f(z), f\left(z_{n}\right)+\rho\left(f_{n}\left(z_{n}\right), f\left(z_{n}\right), a\right)+\rho\left(f\left(z_{n}\right), f(z), a\right)\right.$ (iv)

Again from (iii),

$\rho\left(f\left(z_{n}\right), f(z), a\right) \leq h \max \left\{\rho\left(z_{n}, z, a\right), \rho\left(z_{n}, f\left(z_{n}\right), a\right), \rho(z, f(z), a), \rho\right.$ $\left.\left(z_{n}, f(z), a\right) \rho\left(z, f\left(z_{n}\right), a\right)\right\}$

$\leq \mathrm{h} \max \left\{\rho\left(\mathrm{z}_{\mathrm{n}}, \mathrm{z}, \mathrm{a}\right), \rho\left(\mathrm{z}_{\mathrm{n}}, \mathrm{f}\left(\mathrm{z}_{\mathrm{n}}\right), \mathrm{a}\right)\right\}$

so that

$\rho\left(f_{n}\left(z_{n}\right), f(z), f\left(z_{n}\right)\right)=\rho\left(f(z), f\left(z_{n}\right), z_{n}\right)$

$\leq \mathrm{h} \max \left\{\rho\left(\mathrm{z}_{\mathrm{n}}, \mathrm{z}, \mathrm{z}_{\mathrm{n}}\right), \rho\left(\mathrm{z}_{\mathrm{n}}, \mathrm{f}\left(\mathrm{z}_{\mathrm{n}}\right), \mathrm{z}_{\mathrm{n}}\right)\right\}=0$.

Now (iv) becomes

$\rho\left(z_{n}, z, a\right) \leq \rho\left(f_{n}\left(z_{n}\right), f\left(z_{n}\right), a\right)+h \max \left\{\rho\left(z_{n}, z, a\right), \rho\left(z_{n}\right.\right.$,

$\left.\left.\mathrm{f}\left(\mathrm{z}_{\mathrm{n}}\right), \mathrm{a}\right)\right\}$

which implies

$\rho\left(z_{n}, Z, a\right) \leq \frac{\rho\left(f_{n}\left(z_{n}\right), f\left(z_{n}\right), a\right)}{1-h}<\varepsilon$.

Thus $\mathrm{z}_{\mathrm{n}} \rightarrow \mathrm{z}$.

Our next result generalizes theorem 1.2 of Rhoades [7].

Theorem 4.3 Let $f$ and $g$ be mappings of a complete

$2-$ metric space $X$ into itself satisfying

$\rho(\mathrm{f}(\mathrm{x}), \mathrm{g}(\mathrm{y}), \mathrm{a}) \leq \mathrm{h} \max \{\rho(\mathrm{x}, \mathrm{y}, \mathrm{a}), \rho(\mathrm{x}, \mathrm{f}(\mathrm{x}), \mathrm{a}), \rho(\mathrm{y}, \mathrm{g}(\mathrm{y}), \mathrm{a})$,

$\rho(\mathrm{y}, \mathrm{f}(\mathrm{x}), \mathrm{a}), \rho(\mathrm{x}, \mathrm{g}(\mathrm{y}), \mathrm{a})$, $p\left(x_{s} g(y), a\right)+p(y, f(x), a)$

$\left.\left[\frac{p\left(x_{i} g(y), a\right)+p\left(y_{r} f(x), a\right)}{2}\right]\right\}$ (v)

for all $\mathrm{x}, \mathrm{y} \varepsilon \mathrm{X}, \mathrm{h}$ a fixed constant satisfying $0 \leq \mathrm{h}<1$. Then $\mathrm{f}$ and $\mathrm{g}$ have a common fixed point $\mathrm{z}$ and $(\mathrm{fg})^{\mathrm{n}}\left(\mathrm{x}_{0}\right) \rightarrow \mathrm{z}$ and $(\mathrm{gf})^{\mathrm{n}}\left(\mathrm{x}_{0}\right) \rightarrow \mathrm{z}$ for each $\mathrm{x}_{0} \varepsilon \mathrm{X}$.

Proof: Let $x_{0} \varepsilon X$ and we define $\left\{x_{n}\right\}$ by

$\mathrm{x}_{2 \mathrm{n}+1}=\mathrm{f}\left(\mathrm{x}_{2 \mathrm{n}}\right)$ and $\mathrm{x}_{2 \mathrm{n}+2}=\mathrm{g}\left(\mathrm{x}_{2 \mathrm{n}+1}\right)$

From (vii), we get

$\rho\left(x_{2 n+1}, x_{2 n+2}, a\right)=\rho\left(f\left(x_{2 n}\right), g\left(x_{2 n+1}\right), a\right)$ 


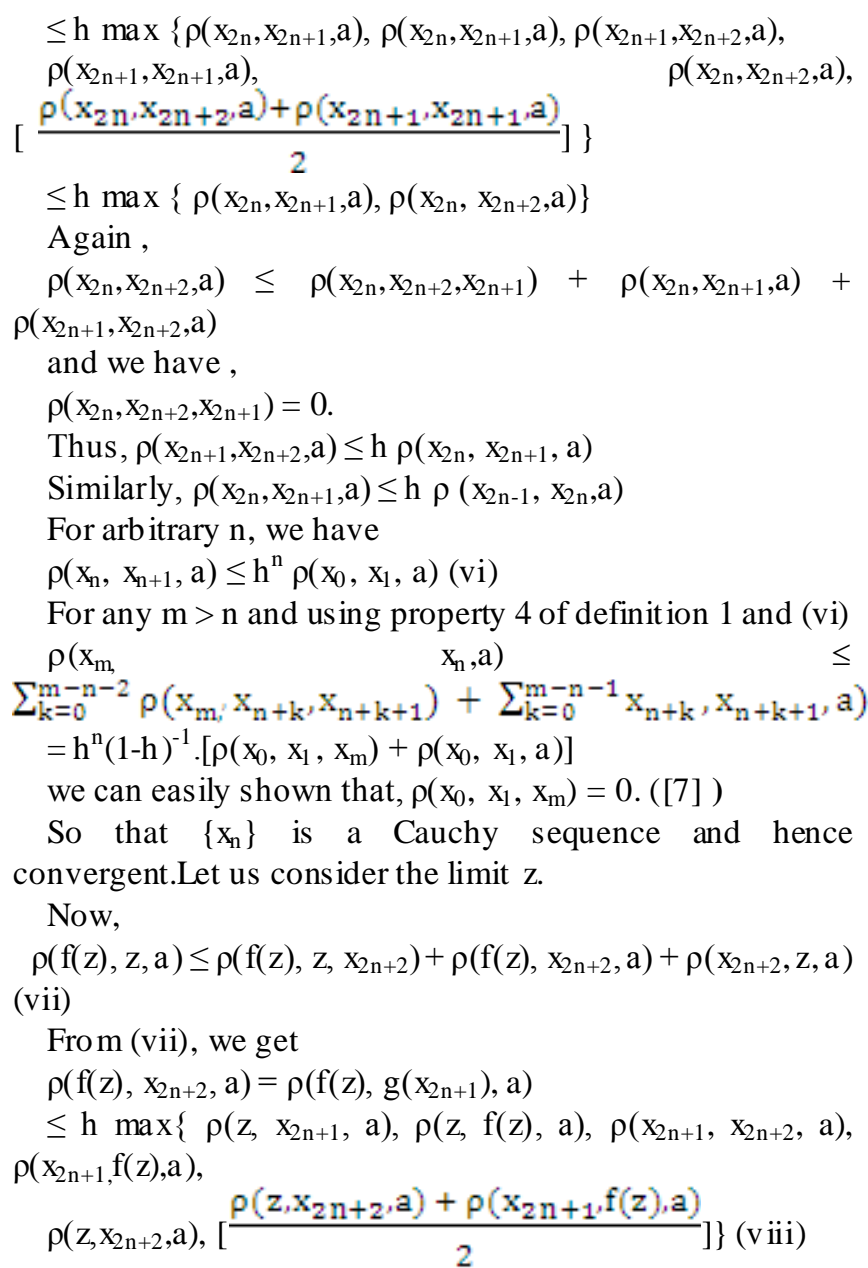

Substituting (vii) into (viii) and taking limit as $n \rightarrow \infty$, we have,

$\rho(f(z), z, a) \leq h \rho(z, f(z), a)$

as $0 \leq \mathrm{h}<1$, we get $\mathrm{z}=\mathrm{f}(\mathrm{z})$.

Therefore, $\mathrm{z}$ is a fixed point of $\mathrm{f}$. Similarly, we can show that $\mathrm{z}$ is also a fixed point of $\mathrm{g}$. For uniqueness, suppose $\mathrm{z}$ and $\mathrm{w}$ are common fixed points of $f$ and $g$.

Now from $(v)$

$\rho(z, w, a)=\rho(f(z), g(w), a)$

$\leq \mathrm{h} \quad \max \{\rho(\mathrm{z}, \quad \mathrm{w}, \quad \mathrm{a}), \quad \rho(\mathrm{z}, \mathrm{f}(\mathrm{z}), \mathrm{a})$, $\rho(w, g(w), a), \rho(z, g(w), a), \rho(w, f(z), a)$,

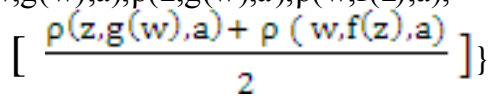

$\leq \mathrm{h} \max \{\rho(\mathrm{z}, \mathrm{w}, \mathrm{a}), 0,0, \rho(\mathrm{z}, \mathrm{w}, \mathrm{a}), \rho(\mathrm{w}, \mathrm{z}, \mathrm{a}), \rho(\mathrm{z}, \mathrm{w}, \mathrm{a})\}$

or, $\rho(z, w, a) \leq h \rho(z, w, a)$,

which implies $z=w$. Thus $z$ is a unique common fixed point of $f$ and $g$.

An extension of theorem 1.3 of Rhoades[7] goes as follows:

Theorem 4.4 Let $X$ be a complete $2-$ metric space, $\left\{f_{n}\right\}, n$ $=1,2, \ldots$. a sequence of mapping $f_{n}: X \rightarrow X$, suppose there exists a sequence of non negative integers $\left\{m_{n}\right\}$ and a number $\mathrm{h}, 0 \leq \mathrm{h}<1$ such that, for all $\mathrm{x}, \mathrm{y} \varepsilon \mathrm{X}$ and every pair $\mathrm{i}, \mathrm{j}, \mathrm{i} \neq \mathrm{j}$ and satisfying

$\rho\left(f_{i}^{m i}(x), f_{j}{ }^{m j}(y), a\right) \leq h \quad \max \quad\{\rho(x, y, a)$, $\rho\left(x, f_{i}^{m i}(x), a\right), \rho\left(y, f_{j}^{m j}(y), a\right), \rho\left(y, f_{i}^{m i}(x), a\right)$, $\rho\left(x, f_{j}^{m j}(y),\left[\rho\left(x, f_{j}^{m j}(y), a\right)+\rho\left(y, f_{i}^{m i}(x), a\right)\right] / 2\right\}(i x)$

Then the mappings $\left\{\mathrm{f}_{\mathrm{n}}\right\}$ have a unique common fixed point.

Proof : Taking $g_{i}=f_{i}^{m i}, i=1,2,3, \ldots$ in (ix), we have $\rho\left(g_{i}(x), g_{j}(y), a\right) \leq h \max \left\{\rho(x, y, a), \rho\left(x, g_{i}(x), a\right), \rho\left(y, g_{j}(y), a\right)\right.$, $\rho\left(y, g_{i}(x), a\right)$,

$\left.\rho\left(x, g_{j}(y), a\right),\left[\frac{\rho\left(x_{v} g_{j}(y) a\right)+\rho\left(y_{i} g_{j}(x)_{a} a\right)}{2}\right]\right\}(x)$

Let us consider $\mathrm{x}_{0} \varepsilon \mathrm{X}$ and we define

$\mathrm{x}_{\mathrm{n}}=\mathrm{g}_{\mathrm{n}}\left(\mathrm{x}_{\mathrm{n}-1}\right), \mathrm{n}=1,2, \ldots$

Now from $(x)$, we get

$\rho\left(x_{n}, x_{n+1}, a\right)=\rho\left(g_{n}\left(x_{n-1}\right), g_{n+1}\left(x_{n}\right), a\right)$

$\leq h \max \left\{\rho\left(x_{n-1}, x_{n}, a\right), \rho\left(x_{n-1}, x_{n}, a\right), \rho\left(x_{n}, x_{n+1}, a\right), \rho\left(x_{n}, x_{n}, a\right)\right.$,

$\rho\left(x_{n-1}, x_{n+1}, a\right)$,

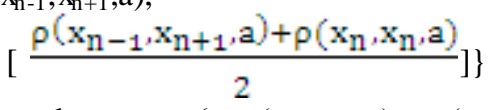

$\leq h \max \left\{\rho\left(x_{n-1}, x_{n}, a\right), \quad \rho\left(x_{n-1}, x_{n}, a\right), \quad \rho\left(x_{n}, x_{n+1}, a\right), 0\right.$,

$\rho\left(x_{n-1}, x_{n+1}, a\right)$,

$\left.\frac{p\left(x_{n-1}, x_{n+1}, a\right)}{2}\right\}$

as in the proof of Theorem 2.3, we led to the conclusion that

$\rho\left(x_{2 n}, x_{2 n+1}, a\right) \leq h \rho\left(x_{2 n-1}, x_{2 n}, a\right)$

and in general

$\rho\left(x_{n}, x_{n+1}, a\right) \leq h^{n} \rho\left(x_{0}, x_{1}, a\right)$.

Therefore, $\left\{x_{n}\right\}$ is Cauchy sequence and converges to a limit $z$. Now we get from $(x)$,

$\rho\left(g_{n}(x), g_{m+1}\left(x_{m}\right), a\right) \leq h \quad \max \left\{\rho\left(z, x_{m}, a\right), \rho\left(z, g_{n}(z), a\right)\right.$, $\rho\left(x_{m}, x_{m+1}, a\right)$,

$\rho\left(x_{m}, g_{n}(z), a\right)$,

$\left.\left[\frac{\rho\left(z_{1}, x_{m+1}, a\right)+\rho\left(x_{m}, g_{n}(z), a\right)}{2}\right]\right\}$

Taking limit as $\mathrm{m} \rightarrow \infty$, we obtain, $\rho\left(g_{n}(z), z, a\right) \leq h \rho\left(g_{n}(z), z, a\right)=h \rho\left(z, g_{n}(z), a\right)$

which implies that $\mathrm{g}_{\mathrm{n}}(\mathrm{z})=\mathrm{z}$, as $0 \leq \mathrm{h}<1$.

For each $n$, we have $f_{n}(z)=f_{n}\left(g_{n}(z)\right)=f_{n}\left(f_{n}{ }^{m n} z\right)$, which shows that $f_{n}(z)$ is a fixed point

of $g_{n}$. Uniqueness of this theorem follows easily from (ix) and by uniqueness, we

have $f_{n}(z)=z$. Hence $z$ is a unique common fixed point of $f_{n}$.

Theorem 4.5 Let $\mathrm{T}$ and $\mathrm{S}$ be two self mappings of a complete metric space $(X, d)$ satisfying $\operatorname{ad}(T x, S y, u)+b d(x, T x, u)+c d(y, S y, u) \leq q \max \{d(x, y, u)$, $\mathrm{d}(\mathrm{x}, \mathrm{T} \mathrm{x}, \mathrm{u}), \mathrm{d}(\mathrm{y}, \mathrm{Sy}, \mathrm{u})\}(\mathrm{xi})$

for $\mathrm{x}, \mathrm{y} \varepsilon \mathrm{X}$ and $\mathrm{u} \varepsilon \mathrm{X}$; $\mathrm{a}+\mathrm{c}>\mathrm{q}$ and $\mathrm{a}>\mathrm{q}$. Then $\mathrm{T}$ and $\mathrm{S}$ have a unique common fixed point.

Proof: Let $\mathrm{x}_{0} \varepsilon \mathrm{X}$ and we define $\left\{\mathrm{x}_{\mathrm{n}}\right\}$ by $\mathrm{x}_{2 \mathrm{n}+1}=\mathrm{Tx}_{2 \mathrm{n}}$ and $\mathrm{x}_{2 \mathrm{n}+2}=\mathrm{Sx}_{2 \mathrm{n}+1}$

Now putting $\mathrm{x}=\mathrm{x}_{2 \mathrm{n}}$ and $\mathrm{y}=\mathrm{x}_{2 \mathrm{n}+1}$ in (xi) we have $\operatorname{ad}\left(\mathrm{Tx}_{2 \mathrm{n}}, \mathrm{Sx}_{2 \mathrm{n}+1}, \mathrm{u}\right)+\mathrm{bd}\left(\mathrm{x}_{2 \mathrm{n}}, \mathrm{Tx}_{2 \mathrm{n}}, \mathrm{u}\right)+\mathrm{cd}\left(\mathrm{x}_{2 \mathrm{n}+1}, \mathrm{Sx}_{2 \mathrm{n}+1}, \mathrm{u}\right)$ $\leq \mathrm{q} \max \left\{\mathrm{d}\left(\mathrm{x}_{2 \mathrm{n}}, \mathrm{x}_{2 \mathrm{n}+1}, \mathrm{u}\right), \mathrm{d}\left(\mathrm{x}_{2 \mathrm{n}}, \mathrm{Tx}_{2 \mathrm{n}}, \mathrm{u}\right), \mathrm{d}\left(\mathrm{x}_{2 \mathrm{n}+1}, \mathrm{Sx}_{2 \mathrm{n}+1}, \mathrm{u}\right)\right\}$ or, $\operatorname{ad}\left(x_{2 n+1}, x_{2 n+2}, u\right)+\operatorname{bd}\left(x_{2 n}, x_{2 n+1}, u\right)+c d\left(x_{2 n+1}, x_{2 n+2}, u\right)$ $\leq \mathrm{q} \max \left\{\mathrm{d}\left(\mathrm{x}_{2 \mathrm{n}}, \mathrm{x}_{2 \mathrm{n}+1}, \mathrm{u}\right), \mathrm{d}\left(\mathrm{x}_{2 \mathrm{n}}, \mathrm{x}_{2 \mathrm{n}+1}, \mathrm{u}\right), \mathrm{d}\left(\mathrm{x}_{2 \mathrm{n}+1}, \mathrm{x}_{2 \mathrm{n}+2}, \mathrm{u}\right)\right\}$ or, $(a+c) d\left(x_{2 n+1}, x_{2 n+2}, u\right)+b d\left(x_{2 n}, x_{2 n+1}, u\right) \leq q d\left(x_{2 n}, x_{2 n+1}\right.$, u) 
or, $d\left(x_{2 n+1}, x_{2 n+2}, u\right) \leq \frac{q-b}{a+c} d\left(x_{2 n}, x_{2 n+1}, u\right)=h d\left(x_{2 n}, x_{2 n+1}\right.$,

$\mathrm{u})$, where $\mathrm{h}=\frac{\mathrm{q}-\mathrm{b}}{\mathrm{a}+\mathrm{c}}$

Similarly, $d\left(x_{2 n}, x_{2 n+1}, u\right) \leq h d\left(x_{2 n-1}, x_{2 n}, u\right)$

Therefore for any arbitrary $n$

$\mathrm{d}\left(\mathrm{x}_{\mathrm{n}}, \mathrm{x}_{\mathrm{n}+1}, \mathrm{u}\right) \leq \mathrm{h}^{\mathrm{n}} \mathrm{d}\left(\mathrm{x}_{0}, \mathrm{x}_{1}, \mathrm{u}\right)(\mathrm{xii})$

From (xii) using the property (4) of definition 1 , we get, for any $m>n$

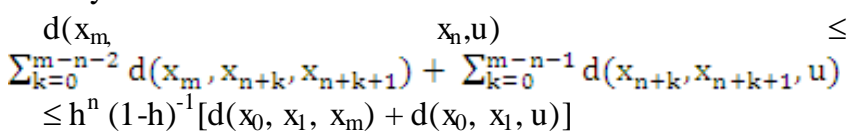

We can easily shown that $\mathrm{d}\left(\mathrm{x}_{0}, \mathrm{x}_{1}, \mathrm{x}_{\mathrm{m}}\right)=0$ ( Rhoades [7] )

So that $\left\{x_{n}\right\}$ is a Cauchy sequence, hence convergent and

$\left\{\mathrm{Tx}_{2 \mathrm{n}}\right\},\left\{\mathrm{Sx}_{2 \mathrm{n}+1}\right\}$ also converge to z. From (xi),

$\operatorname{ad}\left(\mathrm{Tx}_{2 \mathrm{n}}, \mathrm{Sz}, \mathrm{u}\right)+\mathrm{bd}\left(\mathrm{x}_{2 \mathrm{n}}, \mathrm{Tx}_{2 \mathrm{n}}, \mathrm{u}\right)+\mathrm{cd}(\mathrm{z}, \mathrm{Sz}, \mathrm{u})$

$\leq \mathrm{q} \max \left\{\mathrm{d}\left(\mathrm{x}_{2 \mathrm{n}}, \mathrm{z}, \mathrm{u}\right), \mathrm{d}\left(\mathrm{x}_{2 \mathrm{n}}, \mathrm{Tx}_{2 \mathrm{n}}, \mathrm{u}\right), \mathrm{d}(\mathrm{z}, \mathrm{Sz}, \mathrm{u})\right\}$

In the limiting case, we get

$\operatorname{ad}(\mathrm{z}, \mathrm{Sz}, \mathrm{u})+\mathrm{cd}(\mathrm{z}, \mathrm{Sz}, \mathrm{u}) \leq \mathrm{qd}(\mathrm{z}, \mathrm{Sz}, \mathrm{u})$

or, $(a+c-q) d(z, S z, u) \leq 0$.

Therefore, $\mathrm{Sz}=\mathrm{z}$ since $\mathrm{a}+\mathrm{c}>\mathrm{q}$.

Thus $\mathrm{z}$ is a fixed point of S. Similarly we can show that $\mathrm{z}$ is also a fixed point of $T$. Hence $z$ is a common fixed point of $T$ and $\mathrm{S}$.

The uniqueness of the common fixed point can be easily shown by using (xi). This completes the proof of the theorem.

Omitting the term $b d(x, T x, u)$ and $c d(y, S y, u)$ from the left hand side of theorem 4.5 we get the following result as a corollary of the above Theorem.

Cor ollary 4.1 Let the self mappings $\mathrm{T}$ and $\mathrm{S}$ of a co mp lete metric space $(X, d)$ satisfy
$\mathrm{d}(\mathrm{T} x, \mathrm{Sy}, \mathrm{u}) \leq \mathrm{q} \max \{\mathrm{d}(\mathrm{x}, \mathrm{y}, \mathrm{u}), \mathrm{d}(\mathrm{x}, \mathrm{Tx}, \mathrm{u}), \mathrm{d}(\mathrm{y}, \mathrm{Sy}, \mathrm{u})\}$ for $\mathrm{x}, \mathrm{y}, \mathrm{u} \varepsilon \mathrm{X}$. Then $\mathrm{T}$ and $\mathrm{S}$ have a unique common fixed point.

\section{Discussion and Conclusions}

Our results obtained in 2-metric space with inquality condition involving rational terms have generalized the earlier resuls of Gahler, Rhoades, Iseki etc in terms of inquality condition as well as in terms of a pair of mappings and a family of mappings.

\section{REFERENCES}

[1] Ciric, Lj.B. , A generalization of Banach's contraction principle, Proc. Amer. Math.Soc. 45(1974), 267-273.

[2] Gahler, S., 2-metricsche Roume and their topologische structure, Math. Nachr.26(1963), 115-148.

[3] Linear 2- normietre Roume, Math.Nachr.28(1965), 1-43.

[4] Iseki, K, Fixed point theorems in 2-metric space,Math.Sem. Notes,Kobe Univ. 3(1975), 133- 136.

[5] Iseki, K., Sharma, P.L. and Sharma,B.K., Contraction type mappings on 2-metric space, Math.Jap. 21(1976), 67-70.

[6] Khan, M.S., Khan, M.D. , Involutions with fixed points in 2-Banach spaces, Int.J.Math.\& Math.Sci.16 (1993), 429-34.

[7] Rhoades, B.E., Contraction type mappings on a 2-metric space, Math. Nachr. 91(1979), 151-155. 